\title{
On Covers of Cyclic Acts Over Monoids
}

\author{
Mojgan Mahmoudi \\ Department of Mathematics, \\ Shahid Beheshti University, \\ 19839 Tehran, Iran \\ James Renshaw \\ School of Mathematics, \\ University of Southampton, \\ Southampton, SO17 1BJ, England
}

December 10, 2007

\begin{abstract}
In [1] Bican, Bashir and Enochs finally solved a long standing conjecture in module theory that all modules over a unitary ring have a flat cover. The only substantial work on covers of acts over monoids seems to be that of Isbell, Fountain and Kilp ([6], [3], [8]) who only consider projective covers. To our knowledge the situation for flat covers of acts has not been addressed and this paper is an attempt to initiate such a study. We consider almost exclusively covers of cyclic acts and restrict our attention to strongly flat and condition $(P)$ covers. We give a necessary and sufficient condition for the existence of such covers and for a monoid to have the property that all its cyclic right acts have a strongly flat cover (resp. $(P)$-cover). We give numerous classes of monoids that satisfy these conditions and we also show that there are monoids that do not satisfy this condition in the strongly flat case. We give a new necessary and sufficient condition for a cyclic act to have a projective cover and provide a new proof of one of Isbell's classic results concerning projective covers. We show also that condition $(P)$ covers are not unique, unlike the situation for projective covers.
\end{abstract}

\section{Introduction and Preliminaries}

A great deal of work has been done in recent years on various flatness properties of acts over monoids and we refer the reader to [9] for a detailed account of those basic results and definitions that will be needed in this paper. We also refer the reader to [4] for basic definitions and results on semigroup theory. In this paper, unless otherwise stated, $S$ will denote a monoid and $\rho$ a right congruence on $S$ 
so that $S / \rho$ is a cyclic right $S$-act. We shall also deal exclusively with right $S$-acts and simply refer to them as $S$-acts. We shall also consistently write our maps on the left so that $g f$ means $f$ followed by $g$.

After preliminary definitions and results, we define the notion of a cover and a coessential epimorphism in section 2 . The main results here being a description of a coessential epimorphism in terms of congruence classes and a method of constructing covers from left unitary submonoids. In section 3 we define a strongly flat cover and provide a necessary and sufficient condition for a cyclic $S$-act to have a strongly flat cover. We show that not all cyclic acts need have a strongly flat cover and provide a number of interesting classes of monoids which have the property that all their cyclic $S$-acts have strongly flat covers. In section 4 we concentrate on condition $(P)$ covers and achieve similar results to that in section 3. Finally in section 5 we provide a new proof of the classic result on projective covers as well as a new criterion for a cyclic act to have a projective cover. We also show that projective covers are equivalent to the $S$-act counterpart of Enoch's $\mathcal{P}$-covers of modules, where $\mathcal{P}$ is the class of all projective acts.

Recall that unlike the situation for rings and modules, there is a hierarchy of different properties related to the notion of flatness. In particular we have the following sequence of properties, arranged in strictly decreasing order of strength that an act over a monoid may or may not possess.

$$
\begin{gathered}
\text { free } \Longrightarrow \text { projective } \Longrightarrow \text { strongly flat } \Longrightarrow \text { condition }(P) \Longrightarrow \text { flat } \\
\Longrightarrow \text { weakly flat } \Longrightarrow \text { torsion free }
\end{gathered}
$$

Recall that an $S$-act is said

1. to be strongly flat if the tensor functor $A \otimes_{S}$ - preserves pullbacks and equalizers;

2. to satisfy condition $(P)$ if for all $a, a^{\prime} \in A, s, s^{\prime} \in S$ such that $a s=a^{\prime} s^{\prime}$, there exist $a^{\prime \prime} \in A, u, v \in S$ with $a=a^{\prime \prime} u, a^{\prime}=a^{\prime \prime} v$ and $u s=v s^{\prime}$;

3. to satisfy condition $(E)$ if for all $a \in A, s, s^{\prime} \in S$ such that $a s=a s^{\prime}$, there exist $a^{\prime} \in A, u \in S$ with $a=a^{\prime} u$ and $u s=u s^{\prime}$.

It is well known that an act $A$ is strongly flat if and only if it satisfies conditions $(P)$ and $(E)$. We are principally concerned with cyclic $S$-acts here and with the concepts of strong flatness and condition $(P)$ and in these cases there are useful characterisations.

Proposition 1.1 ([9, Theorem III.13.4]) Let $\rho$ be a right congruence on a monoid $S$. Then $S / \rho$ satisfies condition $(P)$ if and only if for all $s, t \in S$, $s \rho t$ implies $u s=v t$ for some $u, v \in S$ with $u \rho 1 \rho v$.

Proposition 1.2 ([3]) Let $\rho$ be a right congruence on a monoid $S$. Then $S / \rho$ is strongly flat if and only if for all $s, t \in S, s \rho t$ implies us = ut for some $u \in S$ with $u \rho 1$. 
A monoid $S$ is said to be right reversible if for all $s, t \in S$ there exists $p, q \in S$ with $p s=q t$. In other words, any two left ideals of $S$ intersect. A monoid $S$ is said to be left collapsible if for all $s, t \in S$ there exists $r \in S$ with $r s=r t$. Clearly left collapsible monoids are right reversible. There are strong connections between right reversible (resp. left collapsible) submonoids of $S$ and condition $(P)$ (resp. strong flatness). We refer the reader to [7], [9] and [11] for more details. A submonoid $T$ of $S$ is said to be left unitary if and only if whenever $t, t s \in T$ then $s \in T$. The following easily proved result will be important.

Proposition 1.3 ([6, 1.3], Cf. [9, Corollary I.4.39]) Let T be a submonoid of a monoid $S$. Then $T=[1]_{\rho}$ for some right congruence $\rho$ if and only if $T$ is a left unitary submonoid of $S$.

In [7] Kilp described a technique to construct a strongly flat cyclic $S$-act from a left collapsible submonoid of $S$. Specifically, if $P \subseteq S$ is a left collapsible submonoid, defining a right congruence on $S$ by

$$
\rho=\{(s, t) \in S \times S: \exists p, q \in P, p s=q t\},
$$

then $S / \rho$ is a strongly flat $S$-act. Notice that we infact only need $P$ to be right reversible in order that the relation $\rho$ is a right congruence. In [11] Renshaw did something similar for right reversible submonoids and cyclic acts satisfying condition $(P)$ but also gave an alternative description of the congruence. We do the same for the strongly flat case here by giving an alternative description of $\rho$ above which will be useful in the proof of Theorem 3.2 below.

Lemma 1.4 (Cf. [11, Lemma 2.2]) Let $S$ be a monoid and $P$ a left collapsible (resp. right reversible) submonoid of $S$. Then $\sigma=(P \times P)^{\sharp}$, the right congruence on $S$ generated by $P \times P$, is such that $P \subseteq[1]_{\sigma},[1]_{\sigma}$ is left collapsible (resp. right reversible) and $S / \sigma$ is strongly flat (resp. satisfies condition $(P)$ ).

Proof. It is clear that $P \subseteq[1]_{\sigma}$. Notice that in either case, $P$ is right reversible and so let $\rho$ be the right congruence on $S$ defined by $s \rho t$ if and only if there exists $p, q \in P$ with $p s=q t$. Notice that $\sigma=\rho$. In fact it is clear that $\sigma \subseteq \rho$ and if $s \rho t$ then there exist $p, q \in P$ with $p s=q t$ and so $s=1 s \sigma p s=q t \sigma 1 t$ and hence $s \sigma t$.

From Proposition 1.1 it then follows that $S / \sigma$ satisfies condition $(P)$. If in addition $P$ is left collapsible then by Proposition $1.2, S / \sigma$ is strongly flat.

\section{Covers and Coessential Epimorphisms}

Definition 2.1 Let $S$ be a monoid and $A$ an $S$-act. We shall say that an $S$-act $C$ together with an $S$-epimorphism $f: C \rightarrow A$ is a cover of $A$ if there is no proper subact $B$ of $C$ such that $\left.f\right|_{B}$ is onto. We shall usually refer to $C$ as the cover. 
It is clear that every $S$-act has a cover, namely itself. However, some acts may have a 'proper' cover and one of the aims of this section is to characterise these for cyclic acts. It is well-known that any cover of a cyclic act must be cyclic ([9, Lemma III.17.19]).

Definition 2.2 Let $S$ be a monoid and $f: C \rightarrow A$ be an $S$-epimorphism. We call $f$ coessential if for each $S$-act $B$ and each $S$-map $g: B \rightarrow C$, if $f g$ is an epimorphism then $g$ is an epimorphism.

It is easy to see that $f: C \rightarrow A$ is a cover of $A$ if and only if it is a coessential epimorphism.

The following is easy to establish.

Lemma 2.3 Let $f: A \rightarrow B$ be a coessential $S$-epimorphism. Then $A$ is cyclic if and only if $B$ is cyclic.

Lemma 2.4 Let $S$ be a monoid and $\sigma, \sigma^{\prime}$ right congruences on $S$. Then $S / \sigma^{\prime}$ is isomorphic to a cyclic subact of $S / \sigma$ if and only if there exists $u \in S$ such that $\left.\sigma^{\prime}=\{(s, t) \in S \times S:(u s, u t) \in \sigma)\right\}$.

Proof. First notice that for any $S$-act $A$ and any $a \in A,\{(s, t) \in S \times S$ : $a s=a t\}$ is a right congruence. So for any right congruence $\sigma$ and $u \in S,\{(s, t) \in$ $S \times S:(u \sigma) s=(u \sigma) t\}$ is a right congruence. If there exists $u \in S$ such that $\left.\sigma^{\prime}=\{(s, t) \in S \times S:(u s, u t) \in \sigma)\right\}$ then $S / \sigma^{\prime} \rightarrow S / \sigma$ given by $s \sigma^{\prime} \mapsto(u s) \sigma$ is a well-defined $S$-monomorphism.

Conversely, let $h: S / \sigma^{\prime} \rightarrow S / \sigma$ be an $S$-monomorphism. Suppose that $h\left(1 \sigma^{\prime}\right)=$ $u \sigma$. Then $h\left(s \sigma^{\prime}\right)=(u s) \sigma$ and since $h$ is well-defined and one-one then $\sigma^{\prime}=$ $\{(s, t) \in S \times S:(u s, u t) \in \sigma)\}$.

Let us denote the $\sigma^{\prime}$ in the previous lemma by $\sigma_{u}$. Note that in some texts (e.g. [9]) $s \sigma_{u} t$ is written as $s(u \sigma) t$. The following is easy to establish:

Lemma 2.5 Let $\sigma$ be a right congruence on $S$ and $u \in S$. Consider the $S-$ monomorphism $h: S / \sigma_{u} \rightarrow S / \sigma$ given by $h\left(s \sigma_{u}\right)=(u s) \sigma$. Then $h$ is onto if and only if $u S \cap[1]_{\sigma} \neq \varnothing$.

We now show that if a cyclic $S$-act has a proper cover then it has a "natural" one.

Lemma 2.6 Let $S$ be a monoid and $\rho$ a right congruence on $S$. If $\sigma$ is a right congruence on $S$ such that $f: S / \sigma \rightarrow S / \rho$ is a coessential epimorphism then there exists $u \in S$ such that $S / \sigma_{u} \cong S / \sigma$ and $f^{\prime}: S / \sigma_{u} \rightarrow S / \rho$ given by $f^{\prime}\left(s \sigma_{u}\right)=s \rho$ is a coessential $S$-epimorphism. In particular, $[1]_{\sigma_{u}} \subseteq[1]_{\rho}$. 
Proof. Since $f: S / \sigma \rightarrow S / \rho$ is onto there exists $u \in S$ with $f(u \sigma)=1 \rho$. Let $\sigma^{\prime}=\sigma_{u}$ so that $S / \sigma^{\prime} \rightarrow S / \sigma$ given by $s \sigma^{\prime} \mapsto(u s) \sigma$ is an $S$-monomorphism whose composite with $f$ is clearly onto. Since $S / \sigma$ is a cover for $S / \rho, S / \sigma^{\prime} \cong S / \sigma$ and $s \sigma^{\prime} \mapsto s \rho$ under the composite epimorphism. It then follows that $[1]_{\sigma^{\prime}} \subseteq$ $[1]_{\rho}$.

We now present one of the main theorems in this paper.

Theorem 2.7 Let $S$ be a monoid and $S / \rho$ a cyclic $S$-act. The map $f: S / \sigma \rightarrow$ $S / \rho$ given by $s \sigma \mapsto s \rho$ is a coessential epimorphism if and only if

$$
\sigma \subseteq \rho \text { and for all } u \in[1]_{\rho}, u S \cap[1]_{\sigma} \neq \varnothing .
$$

Proof. We note first that for any cyclic $S$-act $S / \rho$ and $u \in S$

$$
S / \rho_{u} \cong\left\{[u s]_{\rho}: s \in S\right\}
$$

is an $S$-subact of $S / \rho$. Suppose that $f: S / \sigma \rightarrow S / \rho$ given by $f(s \sigma)=s \rho$ is a coessential epimorphism. Since $f$ is well-defined, $\sigma \subseteq \rho$. Now for any $u \in[1]_{\rho},\left.f\right|_{S / \sigma_{u}}: S / \sigma_{u} \rightarrow S / \rho$ is onto. Since $f$ is coessential, we must have that $[1]_{\sigma} \in S / \sigma_{u}$ and hence $u S \cap[1]_{\sigma} \neq \varnothing$ as required.

Conversely, if the given conditions hold, then clearly $f$ is well-defined. Let $A$ be an $S$-subact of $S / \sigma$ and suppose that $\left.f\right|_{A}$ is onto. Then $1 \rho=f(u \sigma)=u \rho$ for some $u \sigma \in A$. Hence $u \rho 1$ so that $u t \sigma 1$ for some $t \in S$. Consequently, $s \sigma$ uts and so $s \sigma \in A$ for any $s \sigma \in S / \sigma$. Thus $A=S / \sigma$ and $f$ is a cover.

The previous result gives a useful way to check if an epimorphism is coessential. There are occasions when we need to construct covers of cyclic acts and the following result provides a convenient way to do this.

Theorem 2.8 Let $S$ be a monoid and $S / \rho$ a cyclic $S$-act. If $R$ is a submonoid of $[1]_{\rho}$ such that for all $u \in[1]_{\rho}, u S \cap R \neq \varnothing$ then there exists a right congruence $\sigma$ on $S$ such that $R \subseteq[1]_{\sigma}$ and $S / \sigma$ is a cover of $S / \rho$. Moreover, $R=[1]_{\sigma}$ if and only if $R$ is a left unitary submonoid of $S$.

Proof. Define a right congruence $\sigma$ on $S$ by $\sigma=(R \times R)^{\sharp}$. Then clearly $R \subseteq[1]_{\sigma}$. Define a map $f: S / \sigma \rightarrow S / \rho$ by $f(s \sigma)=s \rho$ and note that $f$ is a well-defined $S$-epimorphism. To see this, first notice that if $f$ is well-defined then it is clearly an $S$-map which is onto. Since $R \subseteq[1]_{\rho}$ then $R \times R \subseteq \rho$ and hence $\sigma=(R \times R)^{\sharp} \subseteq \rho$. Since for all $u \in[1]_{\rho}, u S \cap[1]_{\sigma} \neq \varnothing$ then $f$ is coessential by Theorem 2.7 .

By Proposition 1.3 we see that $R=[1]_{\sigma}$ if and only if $R$ is left unitary in $S$.

Corollary 2.9 The cyclic act $S / \sigma$ is a cover of the 1-element $S-$ act $\Theta$ if and only if for all $u \in S$ there exists $s \in S$ with us $\in[1]_{\sigma}$. 
In [9] such acts are referred to as simple.

Theorem 2.10 If $S$ is a monoid and $S / \rho$ is a cyclic $S$-act then the natural map $S \rightarrow S / \rho$ is coessential if and only if $[1]_{\rho}$ is a subgroup of $S$.

Proof. If $S \rightarrow S / \rho$ is coessential then by Theorem 2.7 we know that for all $u \in[1]_{\rho}$ there exists $s \in S$ with $u s=1$. But since $u \rho 1$ then $s \rho u s=1$ and so $s \in[1]_{\rho}$ and the result follows.

Conversely if $[1]_{\rho}$ is a subgroup of $S$ then for all $u \in[1]_{\rho}$ it follows that $u u^{-1} \in$ $\{1\}$ and so from Theorem 2.7, $S \rightarrow S / \rho$ given by $s \mapsto s \rho$ is coessential.

Proposition 2.11 If $S$ is a right simple semigroup with a 1 adjoined and if $\sigma \subseteq \rho$ are right congruences on $S$ with $[1]_{\sigma} \neq\{1\}$ then $S / \sigma \rightarrow S / \rho$ given by $s \sigma \mapsto s \rho$ is a coessential epimorphism.

Proof. This follows from Theorem 2.7 since for all $u \in S \backslash\{1\}, u(S \backslash\{1\})=$ $S \backslash\{1\}$.

Note that if $S / \sigma \rightarrow S / \rho$ is a coessential epimorphism, then taking $R=[1]_{\sigma}$ in Theorem 2.8 gives a right congruence $\psi=(R \times R)^{\sharp}$ on $S$ with $S / \psi \rightarrow S / \rho$ given by $s \psi \mapsto s \rho$ a coessential epimorphism and $[1]_{\psi}=[1]_{\sigma}$. Moreover it is easy to check that $\psi \subseteq \sigma$. They need not be equal however. Suppose, for example, that $T$ is a semigroup and let $S=T^{1}=T \cup\{1\}$ be the monoid obtained by adjoining an extra 1 to $T$ (even if it already has one). If $\sigma \neq \Delta$ is a right congruence on $T$ then setting $\sigma^{1}=\sigma \cup\{(1,1)\}$ gives a right congruence on $S$ and $S / \sigma^{1}$ is a cyclic $S$-act. Note that $[1]_{\sigma^{1}}=\{1\}$ while the corresponding $\psi$ is $\Delta$.

\section{Remark 2.12}

1. It follows from the above that covers of cyclic $S$-acts need not be unique.

2. If $S$ is a group then every cyclic $S$-act has $S$ as a cover and so proper cyclic $S$-acts do not have unique covers. In fact if $S / \sigma \rightarrow S / \rho$ is onto then $S / \sigma$ is trivially a cover of $S / \rho$.

3. If $S$ has a left zero then each $S$-act has a fixed point. Consequently the only cover of $\Theta$ is $\Theta$ itself.

\section{Strongly Flat Covers}

Definition 3.1 Let $S$ be a monoid and $A$ an $S$-act. We shall say that an act $C$ together with an $S$-epimorphism $f: C \rightarrow A$ is a strongly flat cover of $A$ if $C$ is strongly flat and $f$ is coessential. 
Theorem 3.2 Let $S$ be a monoid. Then the cyclic $S$-act $S / \rho$ has a strongly flat cover if and only if $[1]_{\rho}$ contains a left collapsible submonoid $R$ such that for all $u \in[1]_{\rho}, u S \cap R \neq \varnothing$.

Proof. Suppose that $S / \rho$ has a strongly flat cover, $S / \sigma$. Then by Theorem 2.7 we can assume that $R=[1]_{\sigma} \subseteq[1]_{\rho}$ and that for all $u \in[1]_{\rho}, u S \cap R \neq \varnothing$. Moreover, $R$ is left collapsible by Proposition [7, Lemma 2.2].

Conversely, suppose that $R$ is a left collapsible submonoid of $[1]_{\rho}$ such that for all $u \in[1]_{\rho}, u S \cap R \neq \varnothing$. Define a right congruence $\sigma$ on $S$ by $\sigma=(R \times R)^{\sharp}$ as in the proof of Theorem 2.8 so that $S / \sigma$ is a cover of $S / \rho$. Further $S / \sigma$ is strongly flat by Lemma 1.4.

If is worth noting that $R \subseteq[1]_{\sigma}$ and by Proposition $1.3, R=[1]_{\sigma}$ if and only if $R$ is left unitary.

Corollary 3.3 The 1-element $S-$ act $\Theta$ has a strongly flat cover if and only if there exists a left collapsible submonoid $R$ of $S$ such that for all $u \in S$, there exists $s \in S$ with us $\in R$.

The following characterisation of monoids for which all cyclic $S$-acts have a strongly flat cover is then immediate.

Proposition 3.4 If $S$ is a monoid then every cyclic $S$-act has a strongly flat cover if and only if every left unitary submonoid $T$ of $S$ contains a left collapsible submonoid $R$ such that for all $u \in T, u S \cap R \neq \varnothing$.

Theorem 3.5 (1) Let $S$ be a left cancellative monoid. The cyclic $S$-act $S / \rho$ has a strongly flat cover if and only if $[1]_{\rho}$ is a subgroup of $S$ and in this case $S$ is a strongly flat cover of $S / \rho$.

(2) Let $S$ be a monoid and $S / \rho$ a cyclic $S$-act. If $S$ contains a right zero $z$ such that $z \in[1]_{\rho}$ then $S / \rho$ has a strongly flat cover. In particular if $S$ is a right zero semigroup with a 1 adjoined then every cyclic $S$-act has a strongly flat cover.

Proof. (1) Let $S / \sigma$ be a strongly flat cover of $S / \rho$. Then for all $s \sigma t$ there exists $u \in S$ with $u s=u t$. Hence $\sigma=\Delta$ and $S$ is a strongly flat cover of $S / \rho$. By Theorem 2.10, $[1]_{\rho}$ is a subgroup of $S$. The converse follows by Theorem 2.10.

(2) Note that $R=\{1, z\}$ is left collapsible and if $u \in[1]_{\rho}$ then $u z=z \in R$ and so the result follows from Theorem 3.2.

Remark 3.6 In part (1) of Theorem 3.5 notice that the only strongly flat cover of a cyclic $S$-act (assuming that it has one) is then $S$. Notice also that not all cyclic $S$-acts need have a strongly flat cover. For example, if $S=\mathbb{N}$ under multiplication then the only subgroup is $\{1\}$. So no $S / \rho$ for which $[1]_{\rho} \neq\{1\}$ has a strongly flat cover and in particular $\Theta=S / \nabla$ does not have a strongly flat cover. 
The following theorem gives some classes of monoid for which all cyclic $S$-acts have strongly flat covers.

Theorem 3.7 Let $S$ be a periodic monoid such that $E(S)$ is a band and such that every submonoid of $E(S)$ is left collapsible. Then every cyclic $S$-act has a strongly flat cover.

Proof. Let $T$ be a left unitary submonoid of $S$ and let $R=E(T)$. Then for all $u \in T$ there is a power of $u$ that is idempotent and so there is an $s \in S$ with $u s \in R$. Since $R$ is left collapsible the result follows from Proposition 3.4.

Corollary 3.8 Let $S$ be a monoid. Then every cyclic $S$-act has a strongly flat cover if:

(1) $S$ is a periodic monoid and $E(S)$ is a semilattice; or

(2) $S$ is a semilattice with a top element; or

(3) $S$ is a periodic inverse monoid; or

(4) $S$ is a periodic monoid with at most 2 idempotents; or

(5) $S$ is a finite monogenic semigroup with 1 adjoined; or

(6) $S$ is a nil semigroup (every element has a power that is zero) with a 1 adjoined; or

(7) $S$ is left nil semigroup (every element has a power that is left zero) with a 1 adjoined.

Recall from [10] that a monoid $S$ is called right elementary if $S=G \dot{\cup} N$ where $G$ is a group and $N$ is either empty or a right nil semigroup.

Theorem 3.9 Let $S$ be a monoid. Then every cyclic $S$-act has a strongly flat cover if:

(1) $S$ is a regular monoid such that every left unitary submonoid of $S$ is left collapsible; or

(2) $S$ is a left group with a 1 adjoined; or

(3) $S$ is a right group with a 1 adjoined; or

(4) $S$ is elementary.

Proof. (1) Let $T$ be a left unitary submonoid of $S$ and let $u \in T$. Then $u\left(u^{\prime} u\right) \in T$ for all $u^{\prime} \in V(u)$ and so the result follows by Proposition 3.4.

(2) Let $T$ be a left unitary submonoid of $S$. If $T=\{1\}$ then $T$ is left collapsible. Suppose then that $T \neq\{1\}$. For each $1 \neq s \in T$ there is a unique (necessarily idempotent) solution $x=e_{s}$, to the equation $x s=s$. Moreover since $S$ is regular and right cancellative, $e_{s}=s s^{\prime}$ for any $s^{\prime} \in V(s)$. Let $R=\left\{e_{s}: s \in T\right\} \cup\{1\}$ so that $R \subseteq T$. To see this note that $s e_{s} s=s s$ and so $s e_{s}=s$ by right cancellativity. Hence $e_{s} \in T$ since $T$ is left unitary. Since $R$ consists of left zeros, it is left collapsible and for all $1 \neq u \in T, u u^{\prime}=e_{u} \in R$ and the result follows by Proposition 3.4. 
(3) Let $T$ be a left unitary submonoid of $S$. As before we can suppose that $T \neq\{1\}$. For each $1 \neq s \in T$ there is a unique (necessarily idempotent) solution $x=e_{s}$, to the equation $s x=s$. Since $s e_{s}=s$ and since $s \in T$ then $e_{s} \in T$. Let $e$ be one such idempotent and let $R=\{1, e\}$ so that $R \subseteq T$. Then $R$ is left collapsible and for all $1 \neq u \in T$ the equation $u x=e$ has a (unique) solution and so $u S \cap R \neq \varnothing$. The result follows by Proposition 3.4.

(4) Let $T$ be a left unitary submonoid of $S=G \dot{\cup} N$. If there exists $n \in N \cap T$ then $T$ contains a right zero $z$ and so we put $R=\{1, z\}$ which is left collapsible and for all $u \in T, u z=z \in R$. Otherwise $T$ is a subgroup of $S$ and so we let $R=\{1\}$ and again the result follows.

\section{4 (P)-Covers}

Definition 4.1 Let $S$ be a monoid and $A$ an $S$-act. We shall say that an act $C$ together with an $S$-epimorphism $f: C \rightarrow A$ is a $(P)$-cover of $A$ if $C$ satisfies condition $(P)$ and $f$ is coessential.

Clearly every strongly flat cover of $A$ is a $(P)$-cover and in a similar way to Theorem 3.2 we have

Theorem 4.2 Let $S$ be a monoid. Then the cyclic $S$-act $S / \rho$ has a $(P)$-cover if and only if $[1]_{\rho}$ contains a right reversible submonoid $R$ such that for all $u \in[1]_{\rho}, u S \cap R \neq \varnothing$.

Proof. Suppose that $S / \rho$ has a $(P)$-cover, $S / \sigma$. Then by Theorem 2.7 we can assume that $R=[1]_{\sigma} \subseteq[1]_{\rho}$ and that for all $u \in[1]_{\rho}, u S \cap R \neq \varnothing$. Moreover, $R$ is right reversible by Proposition [11, Lemma 2.3].

Conversely, suppose that $R$ is a right reversible submonoid of $[1]_{\rho}$ such that for all $u \in[1]_{\rho}, u S \cap R \neq \varnothing$. Define a right congruence $\sigma$ on $S$ by $\sigma=(R \times R)^{\sharp}$ as in the proof of Theorem 2.8 so that $S / \sigma$ is a cover of $S / \rho$. Further $S / \sigma$ satisfies condition $(P)$ by Lemma 1.4 .

Corollary 4.3 The 1-element $S$-act $\Theta$ has a $(P)$-cover if and only if there exists a right reversible submonoid $R$ of $S$ such that for all $u \in S$, there exists $s \in S$ with $u s \in R$.

Proposition 4.4 If $S$ is a monoid then every cyclic $S$-act has a $(P)$-cover if and only if every left unitary submonoid $T$ of $S$ contains a right reversible submonoid $R$ such that for all $u \in T, u S \cap R \neq \varnothing$.

In contrast to the strongly flat case, since commutative semigroups are necessarily right reversible, we can deduce

Theorem 4.5 Let $S$ be a commutative monoid. Then every cyclic $S$-act has a $(P)$-cover. 
Theorem 4.6 Let $S$ be a monoid. Then every cyclic $S$-act has a $(P)$-cover if:

(1) $S$ is a right simple semigroup with a 1 adjoined; or

(2) $S$ is a periodic left inverse monoid; or

(3) $S$ is a periodic monoid such that $E(S)$ is a band and such that every submonoid of $E(S)$ is right reversible; or

(4) $S$ is a Clifford monoid.

Proof. (1) Let $T$ be a left unitary submonoid of $S$. If $T=\{1\}$ then $T$ is right reversible. Otherwise there exists $s \in T$ and so $R=\langle s\rangle$ is a right reversible submonoid of $T$. It is then clear that for all $u \in T, u S \cap R \neq \varnothing$.

(2) Let $T$ be a left unitary submonoid of $S$. If $T=\{1\}$ then $T$ is right reversible. Otherwise there exists $s \in T$ and so for all $s^{\prime} \in V(s), s^{\prime} s \in T$. Let $R=E(T)$ and note that for all $e, f \in R$, efe $=e f$ and so $R$ is a right reversible submonoid of $T$. Further, for all $u \in T$ there is a power of $u$ that is idempotent and so $u S \cap R \neq \varnothing$.

(3) Let $T$ be a left unitary submonoid of $S$ and let $R=E(T)$. Then for all $u \in T$ there is a power of $u$ that is idempotent and so there is an $s \in S$ with $u s \in R$. Since $R$ is right reversible the result follows from Proposition 4.4.

(4) Let $T$ be a left unitary submonoid of $S$. If $T=\{1\}$ then $T$ is right reversible and for all $u \in T, u S \cap T \neq \varnothing$. If $T$ contains no idempotent other than 1 then notice that if $u \in T$ then $u\left(u^{-1} u\right)=u\left(u u^{-1}\right)=u \in T$ and so since $T$ is left unitary then $u u^{-1}, u^{-1} u \in T$. Hence $u u^{-1}=1$ and so letting $R=\{1\}$ we see that $u S \cap R \neq \varnothing$. Otherwise let $1 \neq e$ be an idempotent in $T$ and let $R=e T$. For all $u \in T, u e=e u \in R$ and so $u S \cap R \neq \varnothing$. Moreover, for all $s, t \in T$ we have

$$
(e t)(e s)=e t t^{-1} \text { tes }=e^{e t e s t^{-1}} t=\left(e^{t s t^{-1}}\right)(e t)
$$

and so $R$ is a right reversible submonoid of $T$.

Proposition 4.7 Let $S$ be a group.

(1) all cyclic $S$-acts $S / \rho \neq S$ have at least two $(P)$-covers.

(2) all cyclic $S$-acts have a unique strongly flat cover.

Proof. (1) First notice that since $S$ is a group then all $S$-acts satisfy condition $(P)$ and so in particular, $S / \rho$ is a $(P)$-cover of itself. However $[1]_{\rho}$ is a subgroup of $S$ and so $S$ is a cover of $S / \rho$ by Theorem 2.10 .

(2) This follows immediately from the remark after Theorem 3.5.

It therefore follows that $(P)$-covers are not unique. In fact if $S$ is an infinite cyclic monoid then there are $(P)$-covers of $\Theta$ of any finite size. To see this let $S=\langle x\rangle$ be an infinite cyclic monoid and let $R=\left\langle x^{n}\right\rangle$ for some $n \in \mathbb{N}$ be a submonoid. Using Corollary 4.3, we see that $\sigma=(R \times R)^{\sharp}$ is such that $S / \sigma$ is a $(P)$-cover of $\Theta$ of size $n$, since $x^{r_{1}} \sigma x^{r_{2}}$ if and only if $r_{1} \equiv r_{2}(\bmod n)$. 
Remark 4.8 It is clear that every strongly flat cover is also a $(P)$-cover and so all of the strongly flat covers considered in section 3 are also $(P)$-covers. However the converse is false. For example by Theorem 4.5 all monogenic semigroups with a 1 adjoined are such that every cyclic $S$-act has a $(P)$-cover. However this is not true for strongly flat covers as the infinite monogenic monoid is left cancellative and so by Theorem 3.5 not all cyclic acts (for example $\Theta$ ) have a strongly flat cover.

\section{Projective Covers and $\mathcal{P}$-Covers}

We present a new shorter proof of [9, Proposition III.17.22] (see also [6, 1.5]) together with a new criterion for projective covers. First recall that $S / \sigma$ is a projective cover of $S / \rho$ if $S / \sigma$ is projective and $S / \sigma \rightarrow S / \rho$ is coessential. Also,

Lemma 5.1 ([9, Corollary III.17.9]) Let $S$ be a monoid. Then the cyclic $S$-act $S / \sigma$ is projective if and only if there exists $e \in E(S)$ with $\sigma=\{(s, t): e s=e t\}$.

Theorem 5.2 Let $S$ be a monoid and $S / \rho$ a cyclic $S$-act. Then the following are equivalent

(1) $S / \rho$ has a projective cover,

(2) there exits a submonoid $R$ of $[1]_{\rho}$ which has a left zero element and for all $u \in[1]_{\rho}, u S \cap R \neq \varnothing$.

(3) the submonoid $[1]_{\rho}$ of $S$ has a minimal right ideal generated by an idempotent.

\section{Proof.}

$(1) \Rightarrow(2)$ Suppose that $S / \sigma$ is a projective cover of $S / \rho$. Then by Theorem 2.7, for all $u \in[1]_{\rho}, u S \cap[1]_{\sigma} \neq \varnothing$. Moreover by Lemma 5.1 there exists $e \in E(s)$ with $\sigma=\{(s, t): e s=e t\}$ and so clearly $e \in[1]_{\sigma}$. If $u \in[1]_{\sigma}$ then $e u=e$ and hence $e$ is a left zero element of $[1]_{\sigma}$.

$(2) \Rightarrow(3)$ Let $z \in R$ be a left zero element of $R$ and consider the right ideal $z[1]_{\rho}$ of $[1]_{\rho}$. If this is not minimal then there exists $u \in[1]_{\rho}$ with $z u[1]_{\rho} \neq z[1]_{\rho}$. By assumption there exists $s \in S$ with $u s \in R \subseteq[1]_{\rho}$ and since $[1]_{\rho}$ is left unitary then $s \in[1]_{\rho}$. Since $z$ is a left zero element of $R, z=z(u s) \in z u[1]_{\rho}$ which is a contradiction.

$(3) \Rightarrow(1)$ Suppose that $e \in E(S)$ is such that $e[1]_{\rho}$ is a minimal right ideal of $[1]_{\rho}$. Define the right congruence $\sigma$ on $S$ by $\sigma=\{(s, t): e s=e t\}$. Then $S / \sigma$ is projective by Lemma 5.1 and the map $s \sigma \mapsto s \rho$ is well defined since if $s \sigma t$ then $e s=e t$. But $e \rho 1$ and so $s \rho e s=e t \rho t$. It is also coessential since if $u \in[1]_{\rho}$ then $e u[1]_{\rho}=e[1]_{\rho}$ by the minimality of $e[1]_{\rho}$ and so $e=e u t$ for some $t \in[1]_{\rho}$. But then $1 \sigma$ ut and so $u S \cap[1]_{\sigma} \neq \varnothing$. Hence the result follows by Theorem 2.7.

Corollary 5.3 The 1-element $S$-act $\Theta$ has a projective cover if and only if there exists a submonoid $R$ of $S$ having a left zero element and such that for all $u \in S$ there exists $s \in S$ with us $\in R$. 
Proposition 5.4 Let $S$ be a monoid. Then all cyclic $S$-acts have a projective cover if and only if for every left unitary submonoid $T$ of $S$ there exists a submonoid $R$ of $T$ having a left zero element and such that for all $u \in T, u S \cap R \neq \varnothing$.

We can see from the preceeding corollary that many of the classes of monoids mentioned in section 3 are such that all cyclic acts have a projective cover. For example

1. a periodic monoid with at most 2 idempotents;

2. a finite monogenic semigroup with a 1 adjoined;

3. a (left) nil semigroup with a 1 adjoined;

4. a right zero semigroup with a 1 adjoined;

5. a left (right) group with a 1 adjoined.

Stenström [12, Corollary 5.4] has proved that every finitely presented strongly flat $S$-act is projective and so for example all cyclic acts over a finite inverse semigroup have a projective cover.

Remark 5.5 It is clear that every projective cover is also a strongly flat cover. However the converse is false. For example by Corollary 3.8 if $S$ is an infinite descending chain with a top element then every cyclic $S$-act has a strongly flat cover and so in particular $\Theta$ has a strongly flat cover. Suppose that $\Theta$ has a projective cover. Then from Corollary 5.3 there is a submonoid $R$ of $S$ containing a left zero element $z$ and such that $u S \cap R \neq \varnothing$ for all $u \in S$. But $z$ is then a minimum element of $R$ and so if we choose $u \in S$ strictly smaller than $z$ then $u s \leq u<z$ for all $s \in S$ and so $u S \cap R=\varnothing$. This is a contradiction and so $\Theta$ does not have a projective cover.

The concept of cover used in [1] is slightly different from that given above.

Definition 5.6 Let $S$ be a monoid, and $A$ be an $S$-act. Let $\mathcal{P}$ be the class of all projective $S$-acts. By a $\mathcal{P}$-precover of $A$ we mean an $S$-map $g: P \rightarrow A$ from some $P \in \mathcal{P}$ such that for every $S$-map $g^{\prime}: P^{\prime} \rightarrow A$, for $P^{\prime} \in \mathcal{P}$, there exists an $S$-map $f: P^{\prime} \rightarrow P$ with $g^{\prime}=g f$.

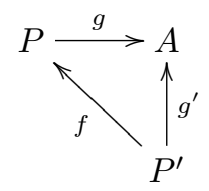

If in addition the precover satisfies the condition that each $S$-map $f: P \rightarrow P$ with $g f=g$ is an isomorphism, then we shall call it a $\mathcal{P}$-cover. 
Theorem 5.7 Cf. [13, Theorem 1.2.12] An $S-$ map $g: P \rightarrow A$, with $P \in \mathcal{P}$, is a projective cover of $A$ if and only if it is a $\mathcal{P}$-cover.

Proof. Let $g: P \rightarrow A$ be a projective cover of $A$. Then $g$ is a $\mathcal{P}$-precover since $P$ is projective. To prove that it is a $\mathcal{P}$-cover, let $f: P \rightarrow P$ be an $S$-map with $g=g f$. Then, $\left.g\right|_{\operatorname{im} f}$ is onto, and so $\operatorname{im} f=P$. Thus $f$ is an epimorphism, and since $P$ is projective, there exists an $S$-map $h: P \rightarrow P$ such that $f h=1_{P}$. So $h$ is a monomorphism and $g h=(g f) h=g(f h)=g$. Thus, $\left.g\right|_{\text {im } h}$ is onto, and hence $\operatorname{im} h=P$. Therefore, $h$ is an epimorphism so $h$ is an isomorphism. Hence $f$ is an isomorphism.

Conversely let $g: P \rightarrow A$ be a $\mathcal{P}$-cover of $A$. Let $F$ be a free act and $f: F \rightarrow A$ be an epimorphism. Since $F$ is free, there exists an $S$-map $h: F \rightarrow P$ such that $g h=f$. Hence $g$ is an epimorphism, since $f$ is. Let $B$ be a subact of $P$ such that $\left.g\right|_{B}$ is an epimorphism. Then since $P$ is a projective, there exists an $S-\operatorname{map} h: P \rightarrow B$ with $\left(\left.g\right|_{B}\right) h=g$. Then we get easily that $g=g \iota h$, where $\iota: B \rightarrow P$ is the inclusion map. Now, by hypothesis, $\iota h$ must be an isomorphism which gives $B=P$.

In the definition of $\mathcal{P}$-(pre)cover, if we replace the class $\mathcal{P}$ of projective acts by another class of acts that is closed under isomorphisms, $\mathcal{X}$ say, then we can easily deduce the following result:

Theorem 5.8 If $g_{1}: X_{1} \rightarrow A$ and $g_{2}: X_{2} \rightarrow A$ are both $\mathcal{X}$-covers of an $S-$ act $A$ then there is an isomorphism $h: X_{1} \rightarrow X_{2}$ such that $g_{2} h=g_{1}$.

So projective covers, being the same as $\mathcal{P}$-covers are unique up to isomorphism. But as we mentioned in Proposition 4.7 this is not true for $(P)$-covers and so if $\mathcal{C} P$ is the class of condition $(P)$ acts then $(P)$-covers and $\mathcal{C} P$-covers are not necessarily the same. We do not know if strongly flat covers are generally unique and so if $\mathcal{S} F$ represents the class of strongly flat covers we do not know if strongly flat covers and $\mathcal{S} F$-covers coincide.

\section{Summary and Open Problems}

It is well known ([9, Proposition III.17.24]) that if an $S$-act $A$ is a union of a strictly ascending chain of cyclic subacts then it does not have a projective cover. This together with Theorem 5.2 allows us to describe projective covers of each act as a coproduct of projective covers of some cyclic acts since the coproducts of coessential epimorphisms are coessential. It would be of interest to extend our results on strongly flat and $(P)$-covers of cyclic acts to acts in general. So we need only consider covers of indecomposable $S$-acts. In particular we can consider locally cyclic $S$-acts. An $S$-act is said to be locally cyclic if every finitely generated subact is contained within a cyclic subact. In other words if for all $x, y \in L$ there exists $z \in L$ with $x, y \in z S$. Suppose that $A$ is a $S$-act and that $L$ is a locally cyclic $S$-act such that $f: L \rightarrow A$ is a coessential epimorphism. Then for all $a, b \in A$ there exists $x, y \in L$ with $a=f(x), b=f(y)$. 
Thus there exists $z \in L, s, t \in S$ with $x=z s, y=z t$ and so $a=f(z) s, b=f(z) t$ and so $A$ is locally cyclic. Thus we see

Theorem 6.1 A non-locally cyclic S-act cannot have a locally cyclic cover.

As mentioned previously we have a strictly decreasing chain of implications relating to covers

$$
\text { projective cover } \Rightarrow \text { strongly flat cover } \Rightarrow(P) \text {-cover. }
$$

There are however monoids for which some of these implications can be reversed. For example, whenever a monoid $S$ has the property that all condition $(P)$ (cyclic) acts are strongly flat then every $(P-)$ cover (of a cyclic act) is also a strongly flat cover. Consequently we can deduce a number of results using the homological classification of monoids. For example,

Theorem 6.2 (1) (Cf [2, Theorem 2.11]) A monoid $S$ is aperiodic if and only if every $(P)$-cover of a cyclic $S$-act is strongly flat.

(2) (Cf [11, Theorem 2.10]) If $S$ is aperiodic and satisfies ACC for cyclic subacts then every $(P)$-cover of any $S$-act is a strongly flat cover.

(3) (Cf [2, Proposition 2.13]) If $S$ is a band then every $(P)$-cover of an $S$-act is strongly flat.

(4) (Cf [5, Corollary 2.14]) If $S$ is nilpotent semigroup with a 1 adjoined then every $(P)$-cover of an $S$-act is a strongly flat cover.

We pose the following open questions for consideration.

1. Is there are monoid $S$ and a cyclic $S$-act $A$ which does not have a $(P)$-cover?

2. Do infinite inverse semigroups have the property that all cyclic $S$-acts have a $(P)$-cover?

3. When does a locally cyclic $S$-act have a strongly flat cover (resp. $(P)$-cover)?

4. What is the criterion for a (locally)cyclic $S$-act to have a flat (resp. weakly flat) cover?

5 . Are strongly flat covers unique?

6. If $\mathcal{F}$ is a class of 'flat' acts (e.g. strongly flat, condition $(P)$, flat, weakly flat) when do $\mathcal{F}$-covers exist?

\section{Acknowledgements}

The authors would like to thank the anonymous referee for some useful comments and suggestions. The first author also thanks the School of Mathematics, University of Southampton, for their kind hospitality during her stay in Southampton in the Spring of 2005, and Shahid Beheshti University for the financial support during her sabbatical leave 


\section{References}

[1] Bican, L., R. EL Bashir and E. Enochs, All modules have flat covers, Bull. London Math. Soc. 33 (2001), 385-390.

[2] Bulman-Fleming, S., Flat and strongly flat S-systems, Comm. in Alg. 20 (1992), 2553-2567.

[3] Fountain, J., Perfect semigroups, Proc. Edinburgh Math. Soc. (2) 20 (1976), 87-93.

[4] Howie, J.M., Fundamentals of Semigroup Theory, London Mathematical Society Monographs, (OUP, 1995).

[5] HuSheng, Q., Strong flatness properties of right $S$-acts satisfying condition (P), Comm. in Alg. 20, No 9, (2002) 4321-4330.

[6] Isbell, J., Perfect monoids, Semigroup Forum 2 (1971), 95-118.

[7] Kilp, Mati, On monoids over which all strongly flat cyclic right acts are projective, Semigroup Forum 52 (1996) 241-245.

[8] Kilp, Mati, Perfect monoids revisited, Semigroup Forum 53 (1996), 225229.

[9] Kilp, Mati, Ulrich Knauer and Alexander V. Mikhalev, Monoids, Acts and Categories, De Gruyter Expositions in Mathematics (29), (Walter de Gruyter, Berlin, New York, 2000).

[10] Renshaw, James and Akbar Golchin, Flat acts that satisfy condition $(P)$, Semigroup Forum 59 (1999), 295-309.

[11] Renshaw, James, Monoids for which condition $(P)$ acts are projective, Semigroup Forum 61 (2000), 46-56.

[12] Stenström, B., Flatness and localisation over monoids, Math. Nachr. 48 (1971), 315-334.

[13] Xu, Jonzhong, Flat Covers of Modules, Lecture Notes in Mathematics, Vol 1634, (Springer-Verlag, 1996). 